SUPPORTING INFORMATION

\title{
Discrete Supracrystalline Heterostructures from Integrative Assembly of Nanocrystals and Porous Organic Cages
}

Mingming Hua,${ }^{\dagger}$ Jinjie Hao,${ }^{\dagger}$ Yanjun Gong, ${ }^{\dagger}$ Fenghua Zhang, ${ }^{\dagger}$ Jingjing Wei,${ }^{\dagger *}$ Zhijie Yang, ${ }^{\dagger *}$ Marie-Paule Pileni

${ }^{\dagger}$ Key Laboratory of Colloid and Interface Chemistry, Ministry of Education, School of Chemistry and Chemical Engineering, Shandong University, Jinan 250100, P.R.

China.

$\$$ Chemistry Department, Sorbonne Université, 4 Place Jussieu, 75005 Paris, France.

*E-mail: *E-mail: weijingjing@sdu.edu.cn and zyangchem@sdu.edu.cn 


\section{SUPPORTING FIGURES}
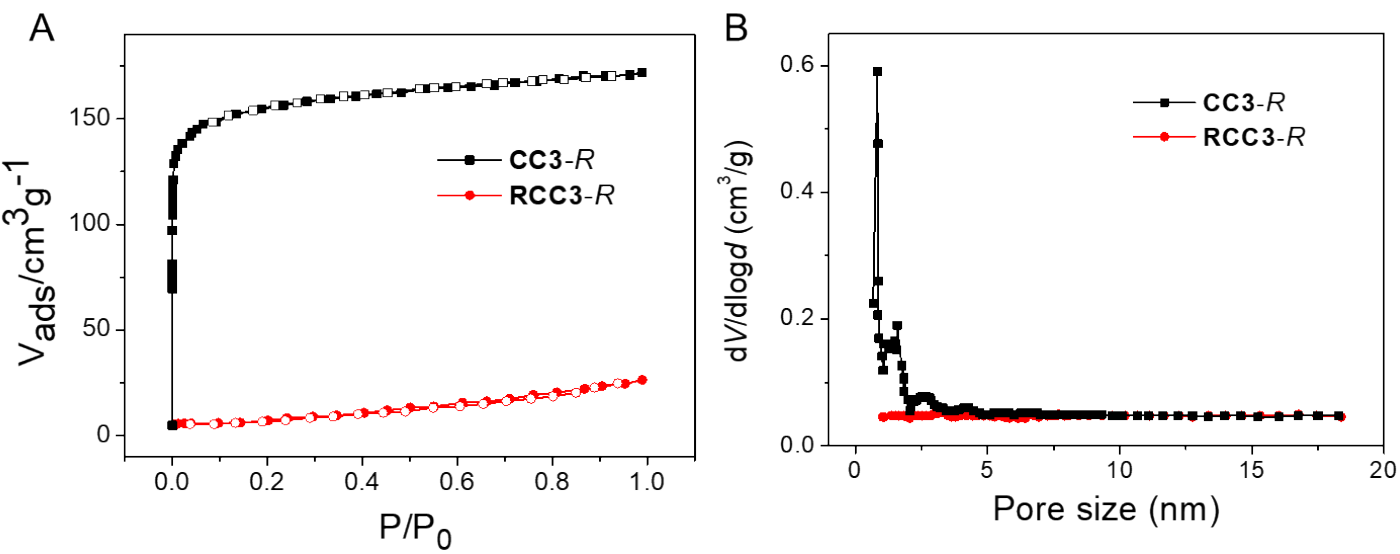

Figure S1. (A) $\mathrm{N}_{2}$ adsorption-desorption curves and (B) the corresponding pore size distributions of CC3- $R$ and $\mathbf{R C C 3}-R$.

A

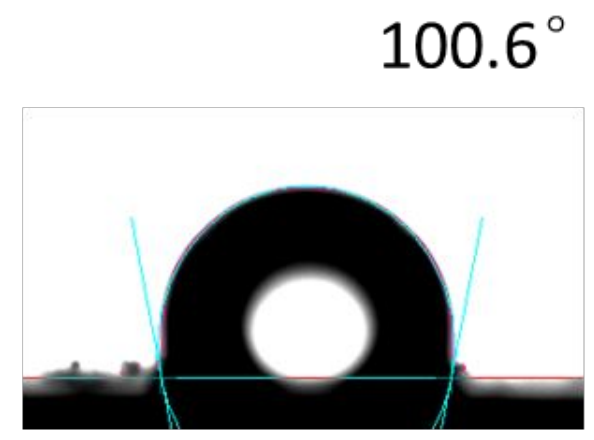

B

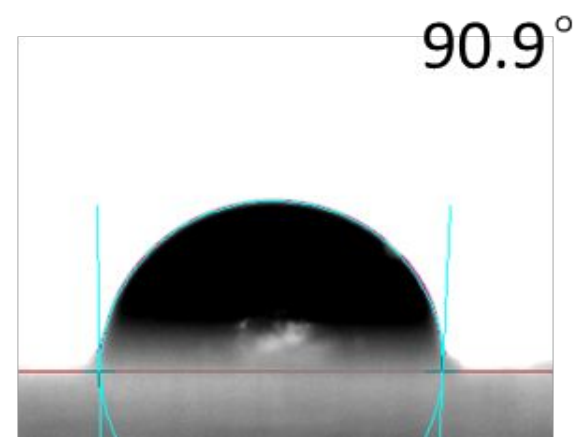

Figure S2. Contact angle measurements for $\mathrm{Au}$ nanocrystals capped with dodecanethiol (A) and porous organic cages CC3- $R$ (B). 


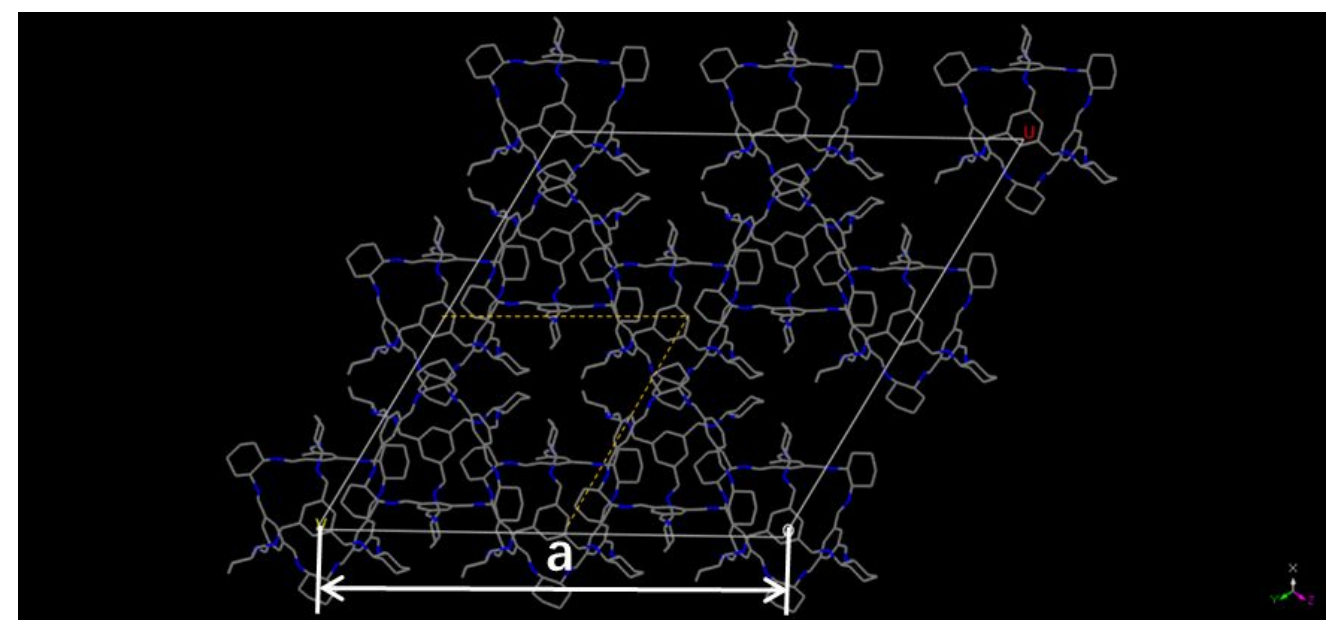

Figure S3. Schematic diagram of (111) crystal plane of CC3- $R$ crystal.

A

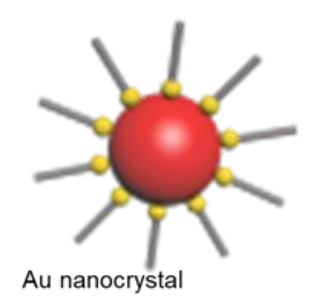

D

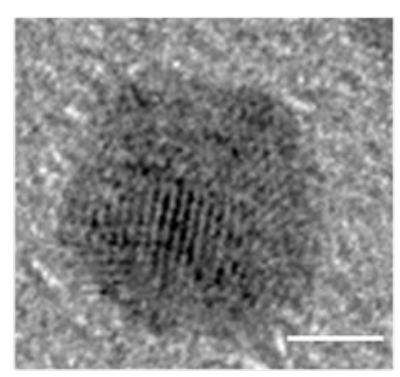

G

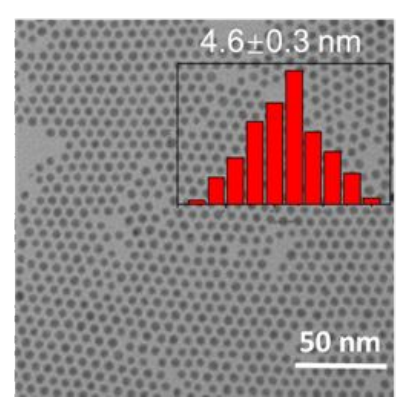

B

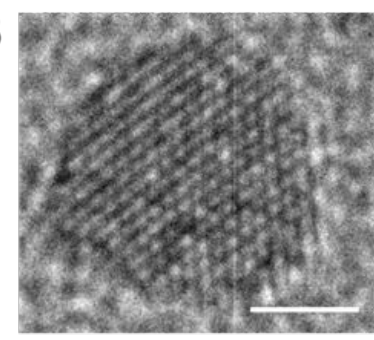

E

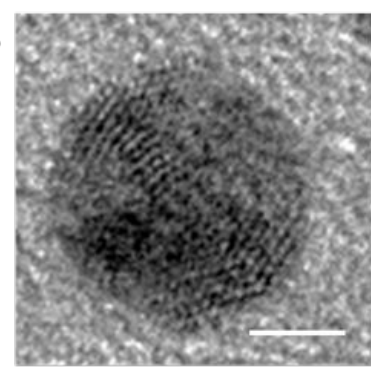

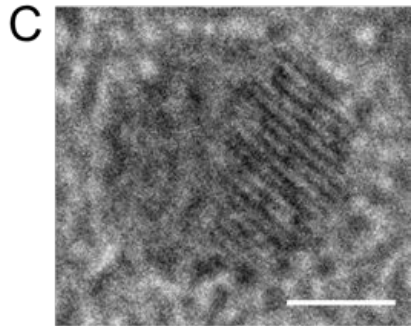

$\mathrm{F}$

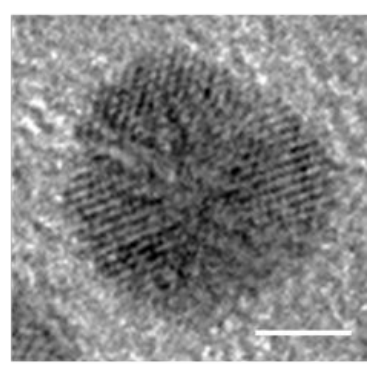

Figure S4. TEM images of quasi-spherical Au nanocrystals (model in A) comprised of various crystallinities: (B) single crystal, (C) poly crystal, (D-F) multiple twinned particle. Scale bars in (B-F) are all $2 \mathrm{~nm}$. (G) low magnification TEM image of $\mathrm{Au}$ nanocrystals. Inset in $(\mathrm{G})$ is the corresponding size histogram. 

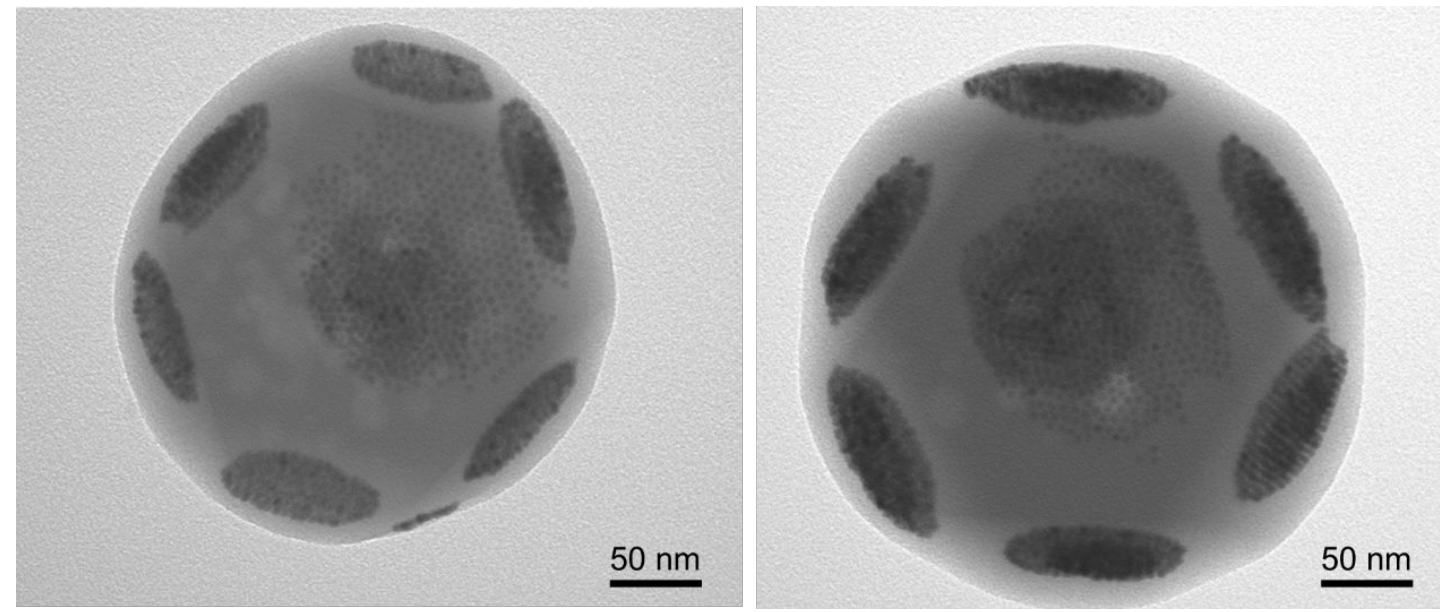

Figure S5. Additional TEM images of the $\mathrm{Au} / \mathbf{C C 3}-R$ assemblies, revealing that each (111) face of the octahedral particles is covered with Au nanocrystal superlattices with a single layer feature.
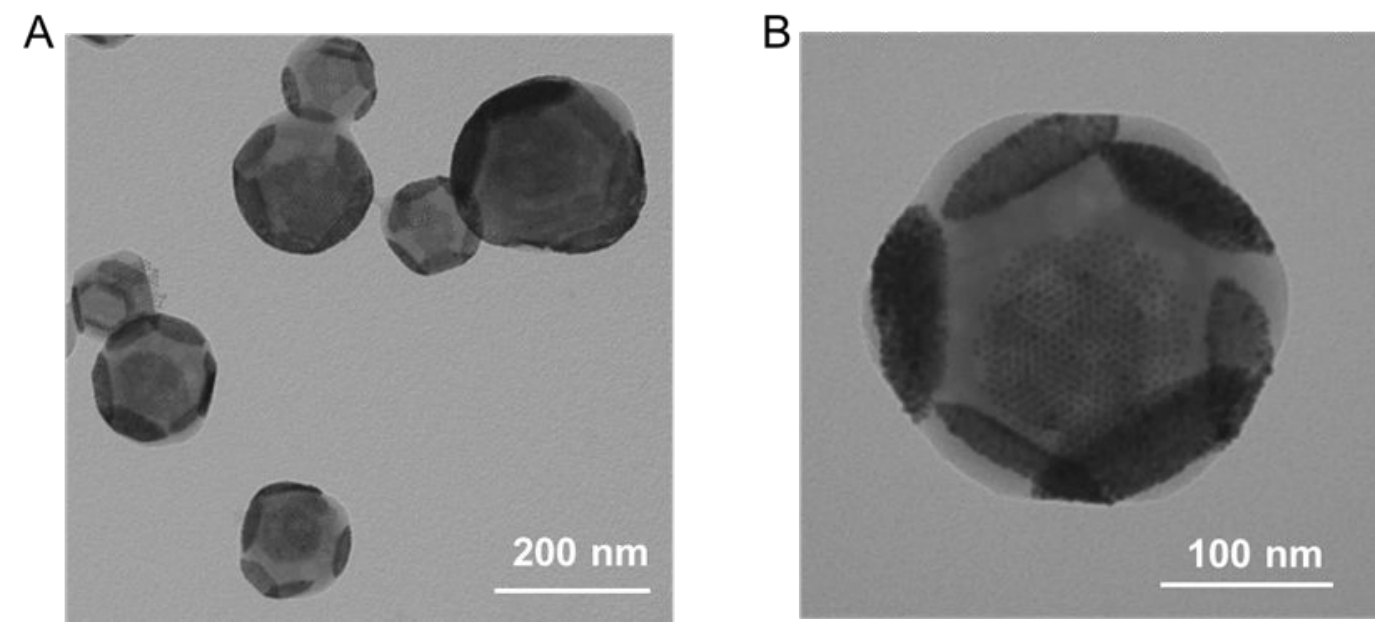

Figure S6. TEM images of $\mathrm{Au} / \mathbf{C C 3}-R$ integrated assemblies suspended in water after six months. 


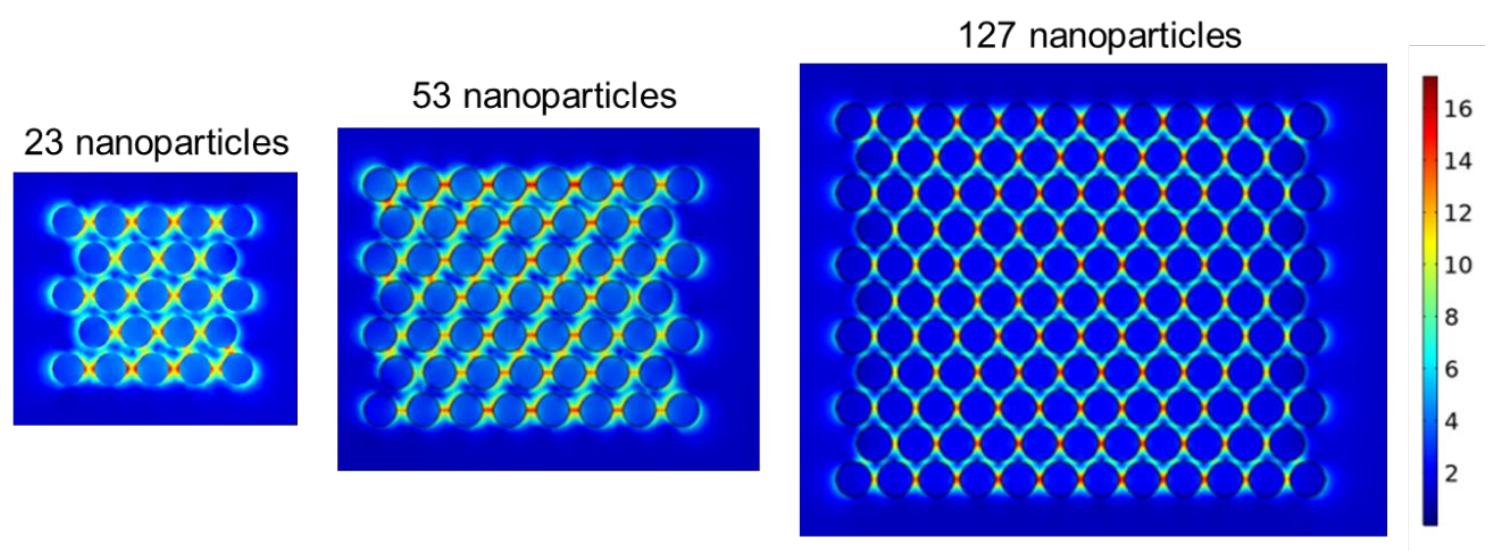

Figure S7. Electromagnetic field of Au nanocrystals self-assembled into 2D arrays with various nanoparticle numbers.

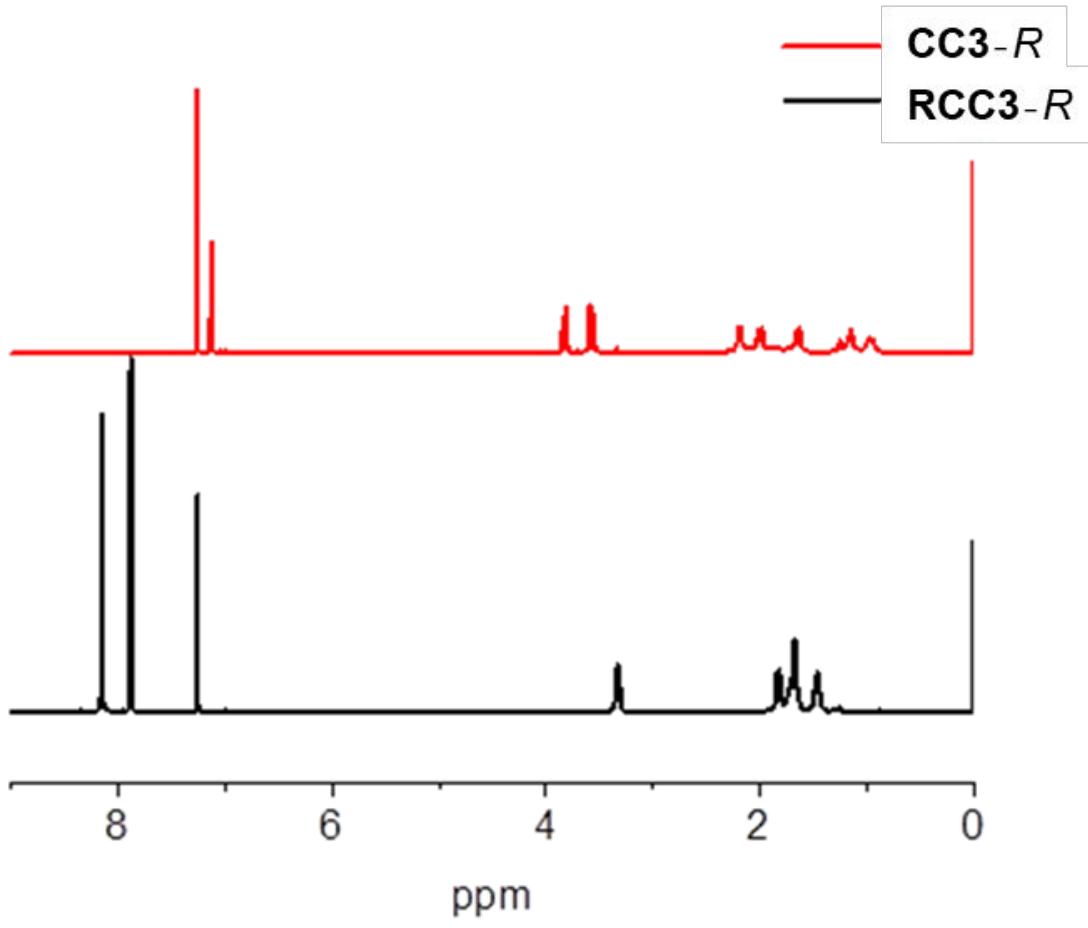

Figure S8. ${ }^{1} \mathrm{H}$ NMR spectra for $\mathbf{C C 3}-R$ and $\mathbf{R C C} 3-R$ dissolved in $\mathrm{CDCl}_{3}$. 


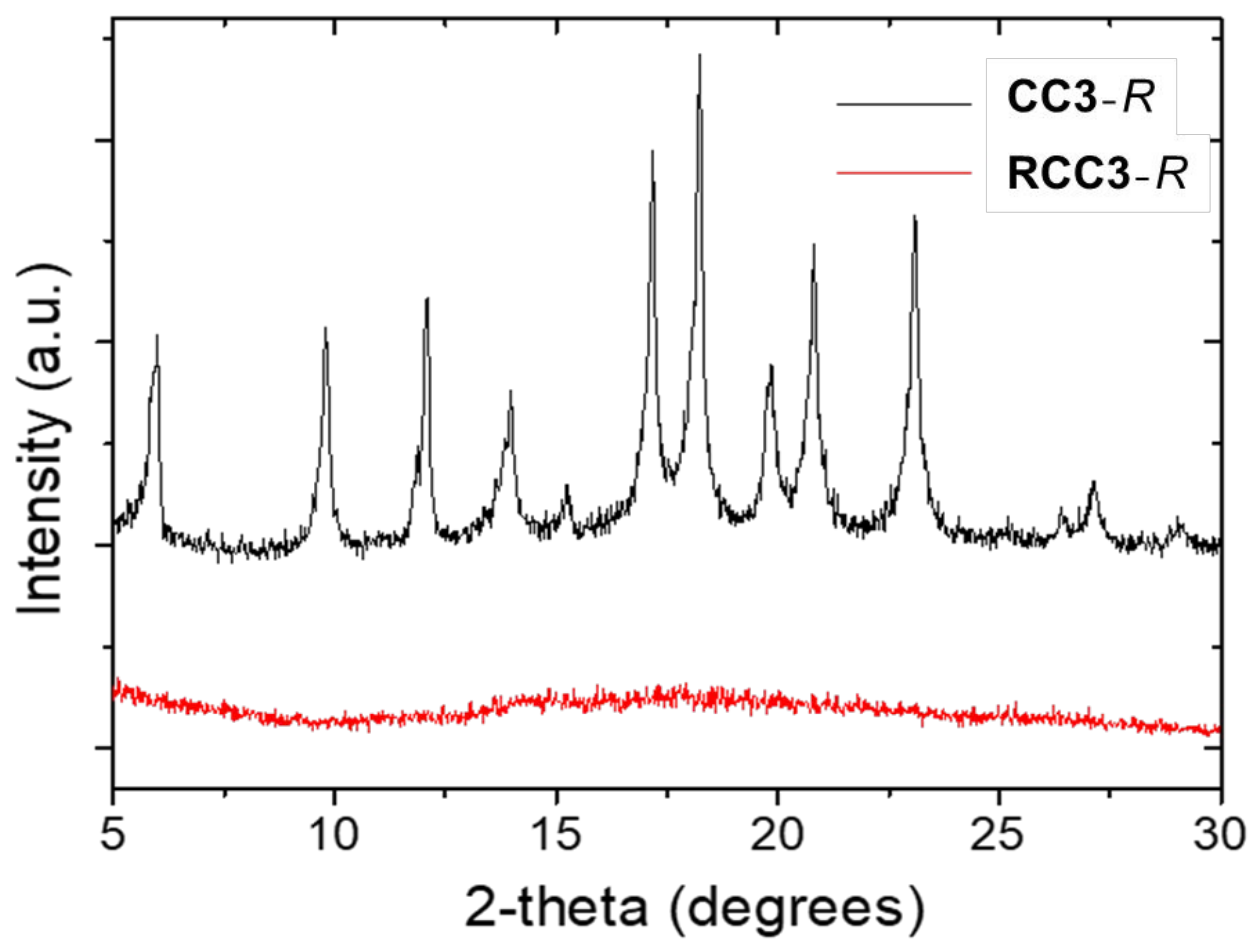

Figure S9. Powder X-ray diffraction patterns of CC3- $R$ and RCC3- $R$ colloidal particles.

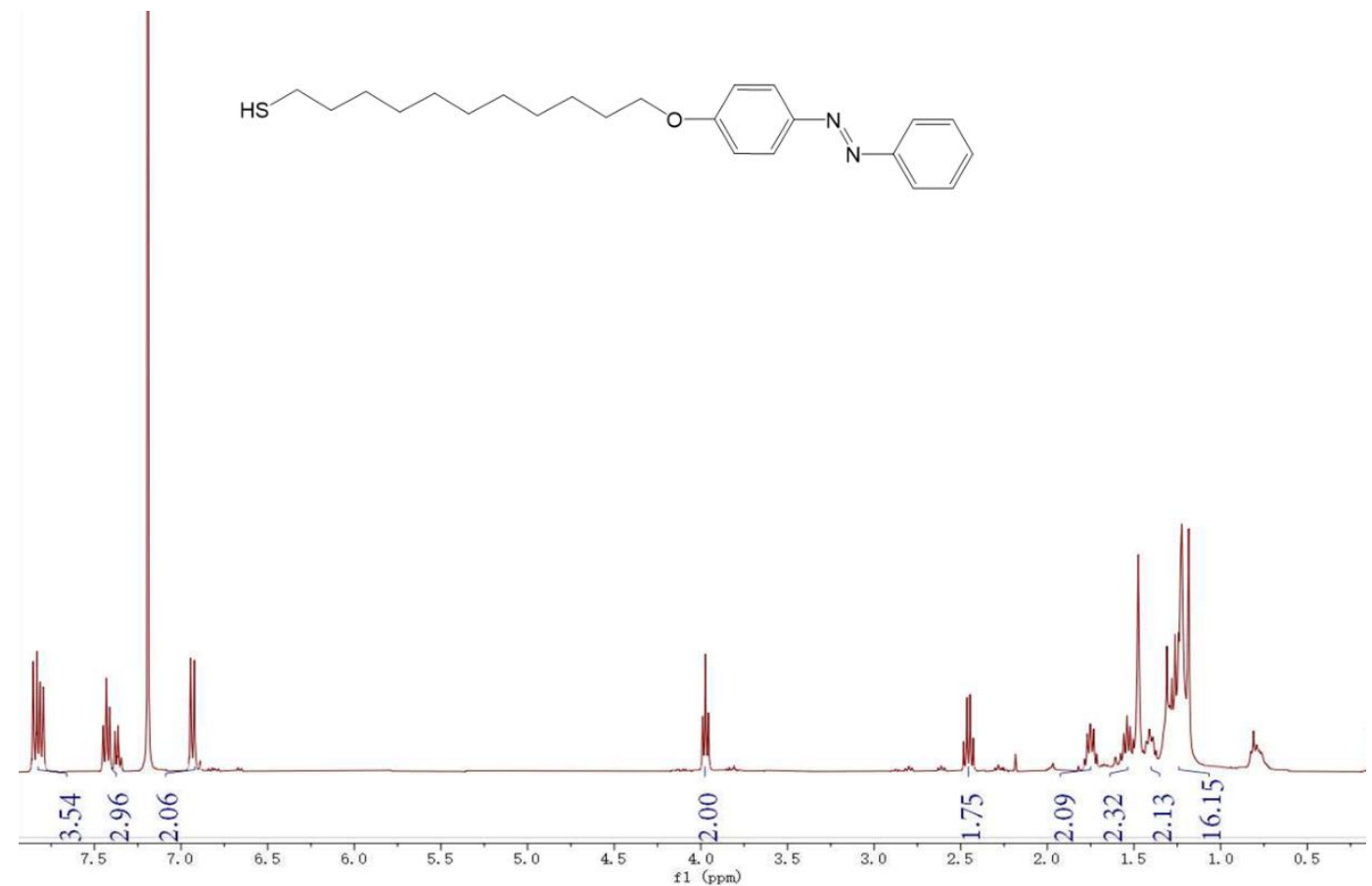

Figure S10. $\quad{ }^{1} \mathrm{H} \quad \mathrm{NMR} \quad$ spectra $\quad\left(400 \quad \mathrm{MHz}, \quad \mathrm{CDCl}_{3}\right)$ of $6-(4-$ (phenyldiazenyl)phenoxy)undecane-1-thiol (AZO-thiol). 

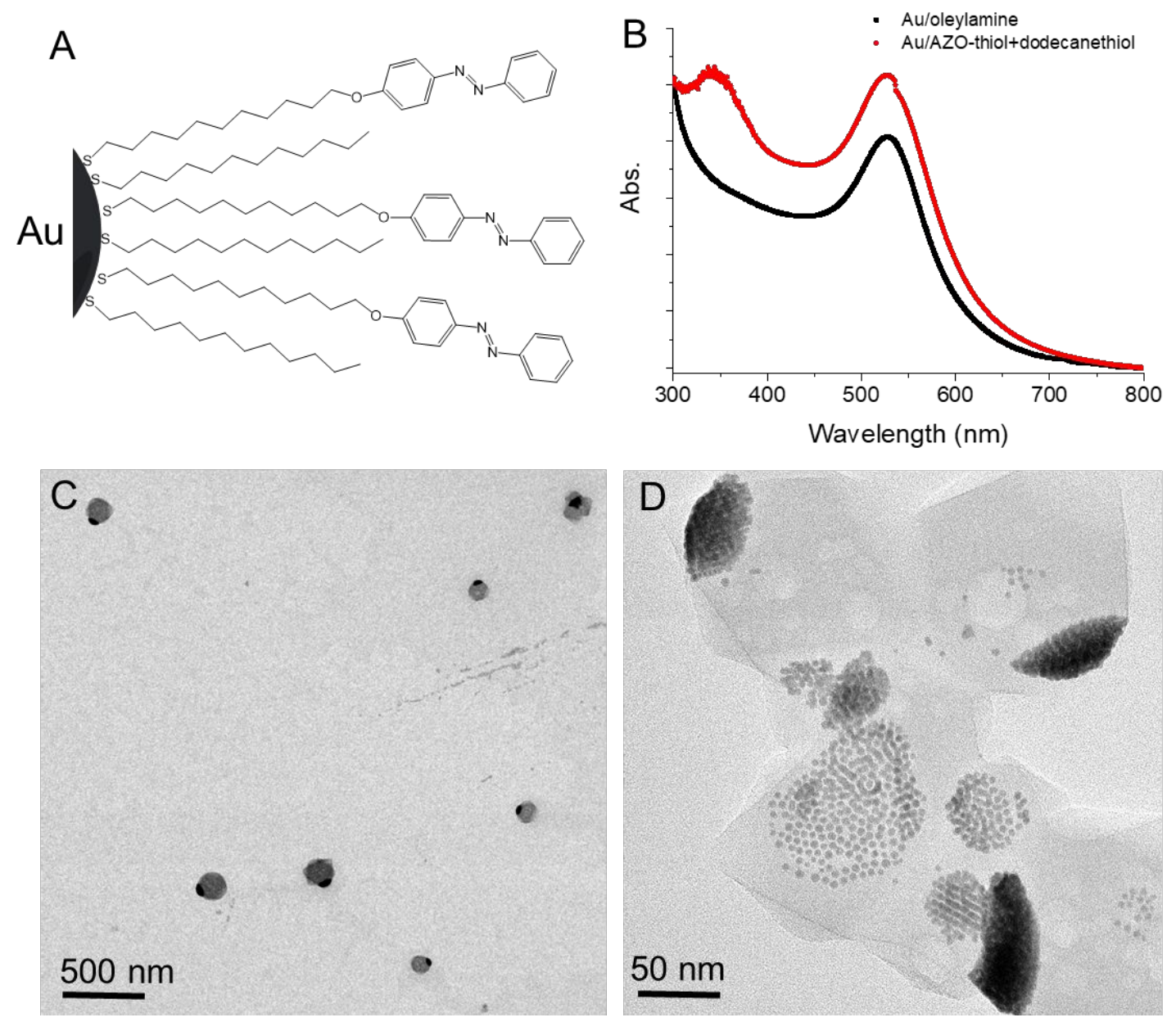

Figure S11. (A) Cartoon illustration of Au nanocrystals coated with a mixed layer of AZO-thiol and dodecanethiol; (B) UV-vis spectra of Au nanocrystals before and after ligand exchange; (C) and (D) are TEM images of assemblies from Au nanocrystals coated AZO-thiol and CC3- $R$. 

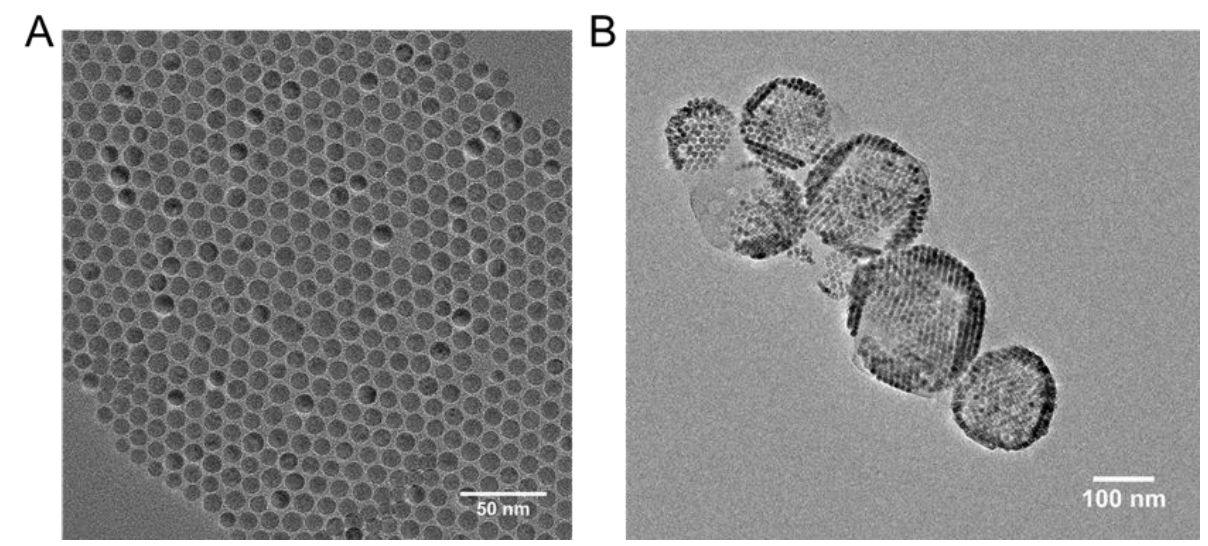

Figure S12. (A) TEM image of spherical $\mathrm{Fe}_{3} \mathrm{O}_{4}$ nanocrystals $(\sim 10 \mathrm{~nm})$. (B) $\mathrm{Fe}_{3} \mathrm{O}_{4} / \mathbf{C C 3}-R$ integrated assemblies.

A
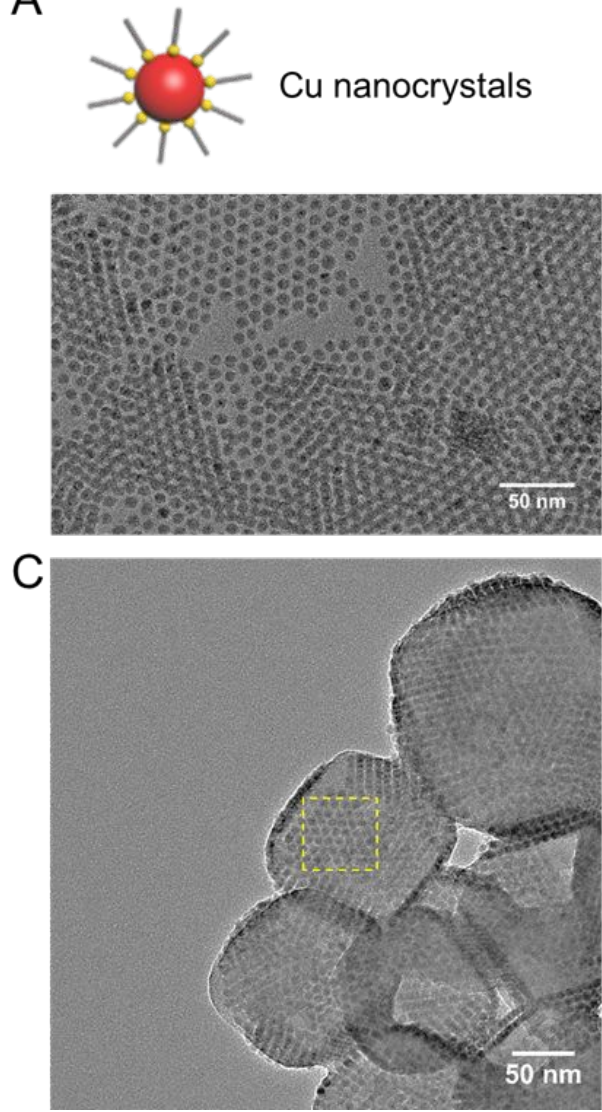

B
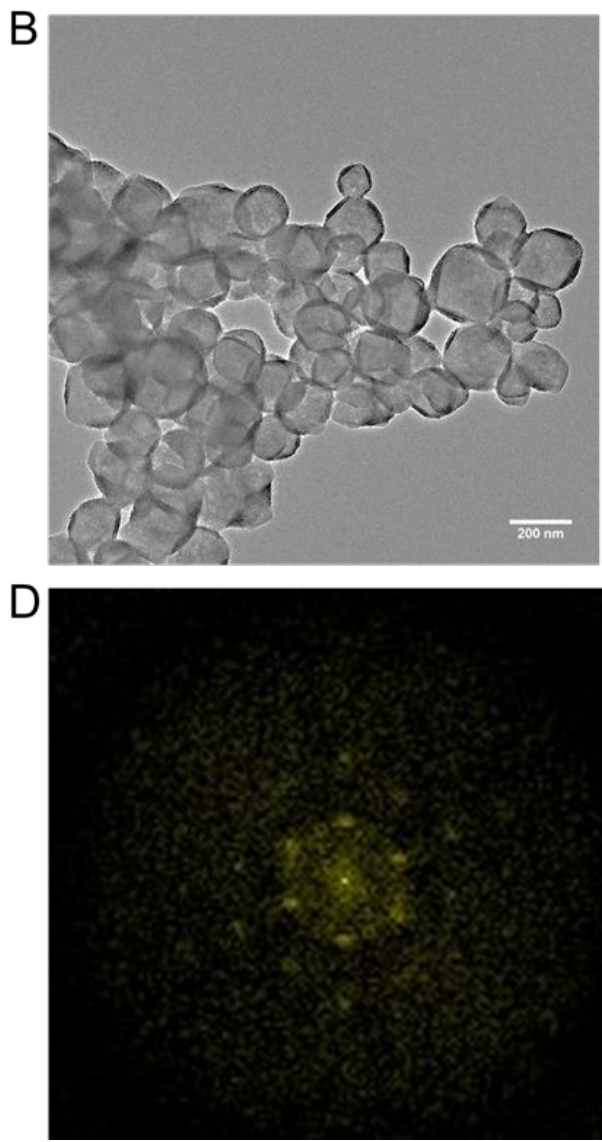

Figure S13. (A) TEM image of $\mathrm{Cu}$ nanocrystals; (B) and (C), TEM images of $\mathrm{Cu} / \mathbf{C C 3}$ $R$ integrated assemblies; (D) Fast Fourier Transform (FFT) pattern in the selected area in $(\mathrm{C})$. 


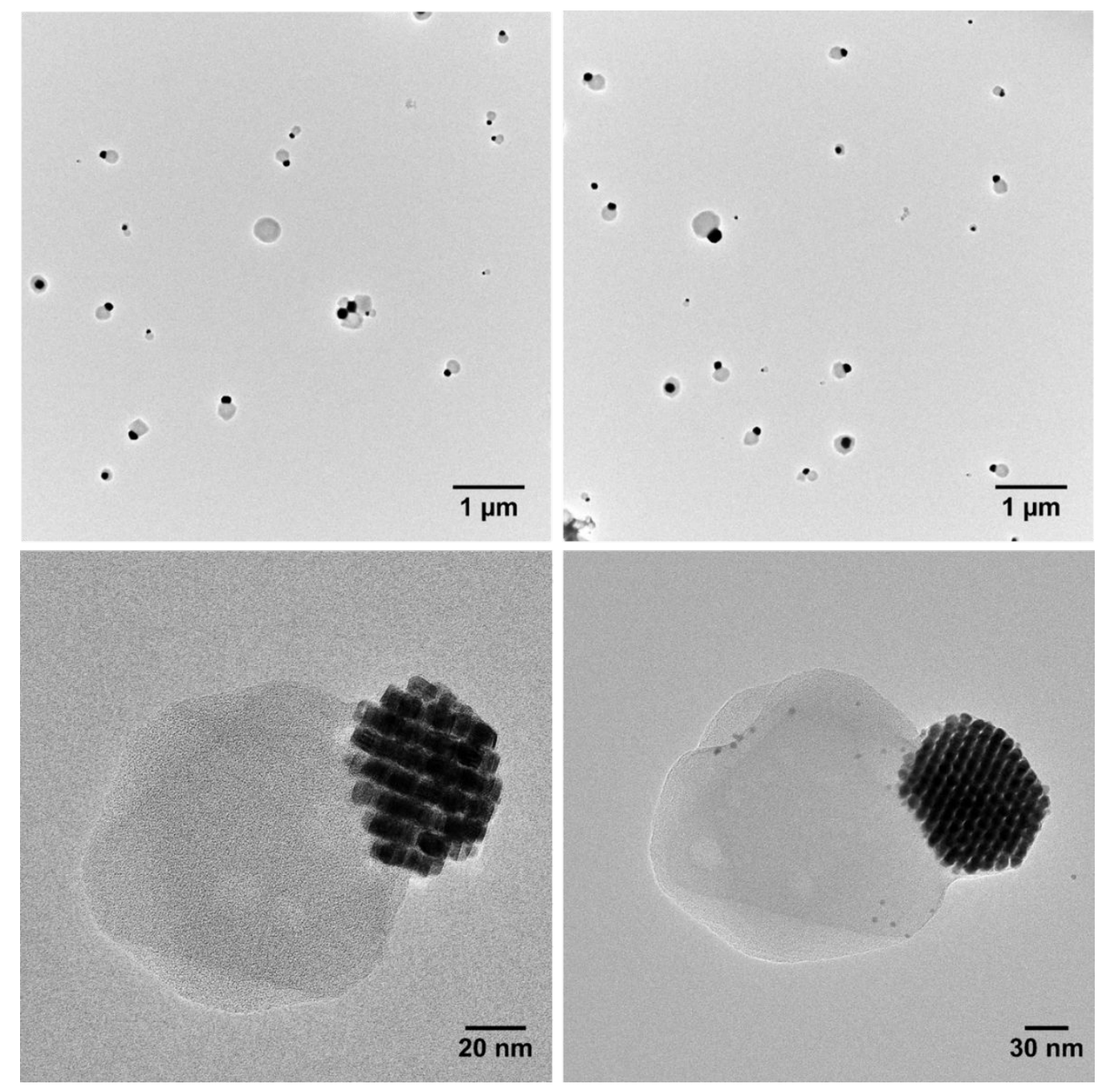

Figure S14. Additional TEM images of the heterodimers from CC3- $R$ cages and $\mathrm{Pt}$ octahedral nanocrystals.

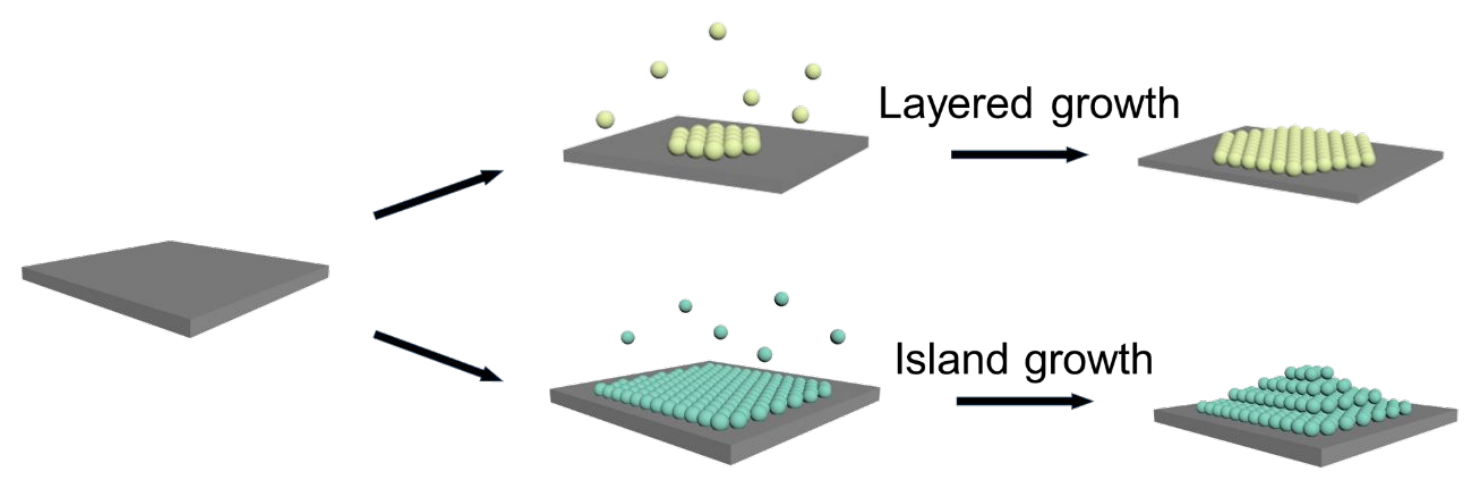

Figure S15. Schematic illustration of atomic epitaxy with two different modes. 

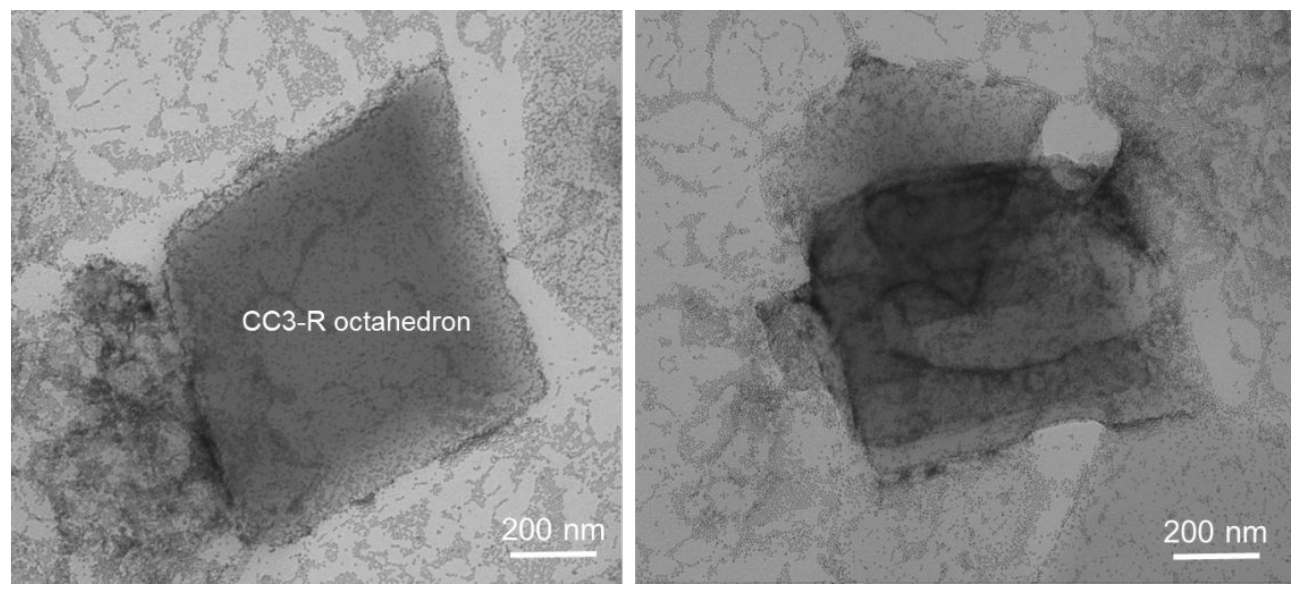

Figure S16. TEM images of the mixture from drop casting of CC3-R and $\mathrm{Au}$ nanocrystals onto a carbon coated TEM grid.

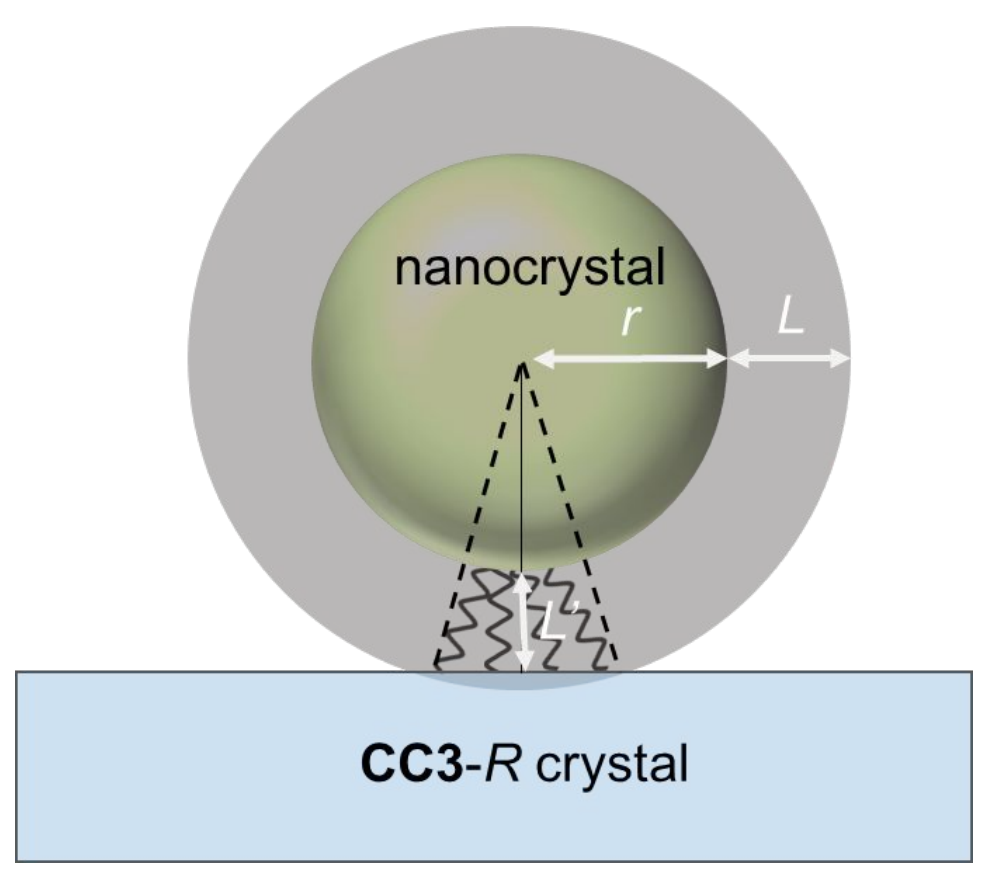

Figure S17. Model used to estimate the contact between a spherical nanocrystal with radius $r$ and the $\mathbf{C C 3}-R$ crystal. 


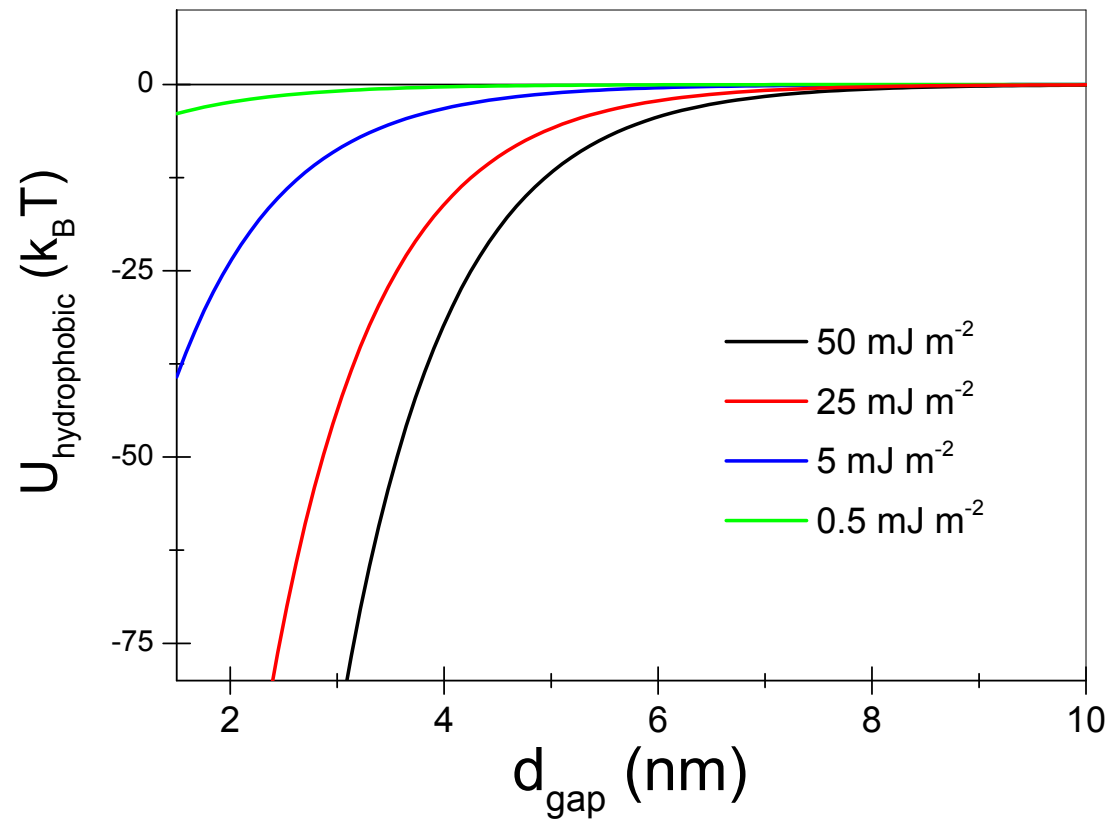

Figure S18. The general hydrophobic effect induced attractive energy between nanocrystals capped with dodecanethiol and CC3- $R$ crystal, depending on the surface tension $\gamma$.

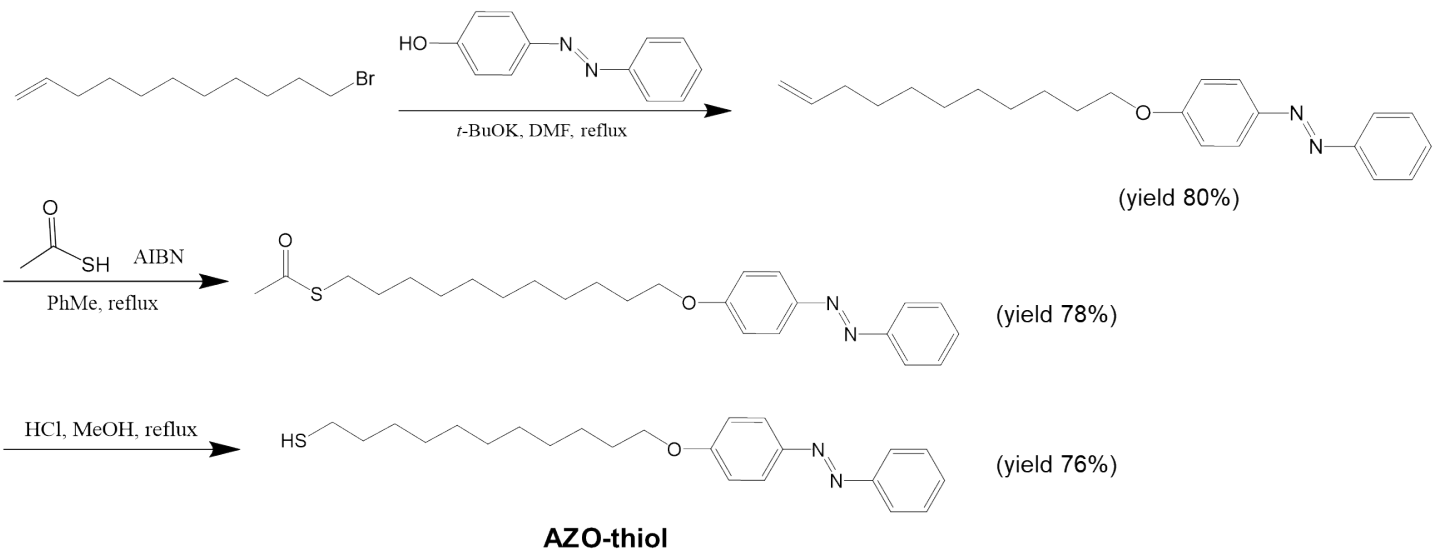

Scheme S1. Synthesis of 6-(4-(phenyldiazenyl)phenoxy)undecane-1-thiol (AZOthiol). 


\section{SUPPORTING TEXT}

\section{Calculation of cavity density on (111) crystal plane of CC3-R octahedral crystal.}

The cavity density of (111) plane of CC3-R crystal is calculated by following formula:

$$
\begin{gathered}
S_{\text {cav }}=a * \frac{\sqrt{3}}{2} a * \frac{1}{2} \\
\rho_{\text {cav }}=\frac{1}{S_{\text {cavity }}}
\end{gathered}
$$

given $\mathrm{a}=17.5362 \AA$, we obtain $S_{\text {cav }}=0.7541 \mathrm{~nm}^{2}, \rho_{\text {cav }}=1.3261 \mathrm{~nm}^{-2}$.

COMSOL finite element method (FEM) simulations. The absorption cross sections were calculated using COMSOL Multiphysics finite-element based software. The 'Wave optics' module was utilized to calculate the scattered field resulting from a plane wave incident on a model 3-D nanocrystal. The plane wave was defined as: $E=E_{0}$ $e^{-i k x}$ and was polarized in the z-direction. Hexagonal-close-packed nanocrystal superlattice were defined and the free space wavelength range from $400 \mathrm{~nm}$ to $800 \mathrm{~nm}$ was simulated. Three different quantities of particles (23, 53 and 127) were simulated. The optical data for Au were taken from COMSOL's Optical Materials database ${ }^{1}$ and a region of water was defined around the particle by setting the real part of the dielectric function equal to 1.33 . The width of the region of water around the particle was equal to half the wavelength of the incident plane wave and a perfectly matched layer (PML) was constructed to act as an absorber of the scattered field. Calculating the absorption cross sections of nanoparticles is important for determining the LSPR wavelength. The absorption cross section as a function of wavelength was calculated by integrating the 
resistive heating losses over the volume of the nanoparticles. The LSPR peak position for the Au nanocrystal is around $540 \mathrm{~nm}$.

Estimation the attractive energy from the host guest interactions between CC3-R (111) plane and Au nanocrystals. When a single Au nanocrystal $(\mathrm{r}=2.3 \mathrm{~nm}, \mathrm{~L}=1.5 \mathrm{~nm})$ contact with (111) crystal plane of the CC3- $R$ crystal (Figure S17). The penetration depth is assumed to be $\sim 0.36 \mathrm{~nm}$, which is estimated from the optimized structure from DFT calculation. Therefore, the contact front area $\left(S_{\text {contact }}\right)$ is around $8.2 \mathrm{~nm}^{2}$. Considering the fact that a much higher grafting density of dodecanethiol $\left(p_{S H}\right)$ on gold surface $\left(\sim 4.6 \mathrm{~nm}^{-2}\right)$ than that of the cavity on (111) plane of $\mathbf{C C 3}-R$ crystal $\left(p_{c a v} \sim 1.3\right.$ $\mathrm{nm}^{-2}$ ), the $N_{\mathrm{HG}}$ is mainly determined by $p_{\text {cav }}$, and the $N_{\mathrm{HG}}$ is estimated to be $\sim 11$. In such a scenario, $\sim 70 \%$ of the alkyl chains contacted with $\mathbf{C C 3}-R$ crystal can include into the cavities. We note that the above rough calculation did not consider the distortion of the cavity of CC3- $R$, which could affect the host-guest interactions between cavity and alkyl chain.

The contribution from general hydrophobic effect. The general hydrophobic effect was previously described by Israelachvili et $a{ }^{2}{ }^{2}$ for stressed surfactant bilayers. The interaction potential was described by the formula as follows:

$$
U=-2 \gamma\left(s-s_{0}\right) e^{-\frac{d_{g a p}}{d_{0}}}
$$

Where $U$ is the energy per molecule, $\gamma$ is the surface tension, $s$ and $s_{0}$ are the area per molecule and the equilibrium area per molecule, respectively, $d_{g a p}$ and $d_{0}$ are the intermolecular distance and the hydrophobic decay length, respectively. Hence the hydrophobic potential ( $W_{\text {hydrophobic }}$ ) of the energy per unit area can read as follows: 


$$
W_{\text {hydrophobic }}=-2 \gamma\left(1-\frac{s_{0}}{s}\right) e^{-\frac{d_{\text {gap }}}{d_{0}}}
$$

For the curved surface with radius $r$ and the ligand length $L$, the energy can be described:

$$
U_{\text {hydrophobic }}=-4 \pi r d_{0} \gamma\left(1-\frac{s_{0}}{s}\right) e^{-\frac{\left(d_{g a p}-L\right)}{d_{0}}}
$$

The decay length for the hydrophobic force $d_{0}=1 \mathrm{~nm}$ as indicated by previous measurements, and $s_{0}$ equals to 0 for the fully hydrophobic surface. In our case, the surface tension $\gamma$ is impossible to be determined from an evaporating mixture of chloroform/water. We found that the attractive energy from general hydrophobic effect varies (Figure S18), depending on the specific surface tension $\gamma$. The maximum of $\gamma$ is derived from that of water/dodecane (The surface of Au nanocrystals is capped with dodecanethiol) after the total removal of chloroform. We claim that this general hydrophobic effect contributes to the colloidal stability of the Au/CC3- $R$ (including $\mathrm{Pt} / \mathbf{C C} 3-R$ and $\mathrm{Au} / \mathbf{R C C 3}-R$ ) heterostructures dispersed in water.

\section{SUPPORTING REFERENCES}

1. Rakić, A. D.; Djurišić, A. B.; Elazar, J. M.; Majewski, M. L. Optical Properties of Metallic Films for Vertical-Cavity Optoelectronic Devices. Appl. Opt. 1998, $37,5271-5283$.

2. Donaldon, S.H.; Lee C. T. Jr.; Chmelka, B. F.; Israelachvili J. N. General Hydrophobic Interaction Potential for Surfactant/Lipid Bilayers from Direct Force Measurements between Light-Modulated Bilayers. Proc. Natl. Acad. Sci. U.S.A. 2011, 108, 15699-15704. 